\section{Bud Development in Response to Night-breaking Treatment in the Noninductive Period in Red Pitaya (Hylocereus sp.)}

\author{
Yi-Lu Jiang \\ Department of Horticulture, National Chiayi University, No. 300 Syuefu \\ Road, Chiayi City 60004, Taiwan
}

\author{
Yuan-Yin Liao, Meng-Tzu Lin, and Wen-Ju Yang ${ }^{1}$ \\ Department of Horticulture and Landscape Architecture, National Taiwan \\ University, No. 1, Sector 4, Roosevelt Road, Taipei 106, Taiwan
}

Additional index words. daylength, floral induction, floral evocation, long-day plant, photoperiod

\begin{abstract}
Off-season flowering in red pitaya (Hylocereus sp.), a long-day plant, can be achieved using night-breaking (NB) treatment. Among the stages of bud development, stage 0 referred to induced but not yet differentiate any bracteole and stage 3 was the stage right before emerging floral buds and the bracteole differentiation was completed. Unlike floral bud emergence, bracteole differentiation was independent of the daylength and strongly influenced by the environmental temperature. The buds of higher stages were more effective in response to NB treatment and more sensitive to chilling injury (CI). Consequently, off-season flowers in autumn and winter trials were derived mainly from stage 2 and 3 buds and from stage 0 and 1 buds, respectively. In southern Taiwan, low night temperature between $10 \mathrm{Jan}$. and $7 \mathrm{Feb} .2011$ may be the major factor, which delay bud development in off-season production. Therefore, we conducted a heating experiment in winter off-season production to proof our hypothesis and concluded that NB treatment should be applied along with night temperature elevation or after midFebruary when the minimum night temperature is increasing.
\end{abstract}

Red pitaya (Hylocereus sp.), a climbing cactus, was introduced into Taiwan as a fruit crop during $1980 \mathrm{~s}$ and has increased in popularity (Chang, 2003; Hsu, 2004; Jiang, 2005; Jiang et al., 2011; Yen and Chang, 1997). In Taiwan, the most commonly grown red pitaya varieties are selected from crosses between Hylocereus undatus and Hylocereus sp. Pitaya is a long-day plant, which flowers in several flushes between May and October in the northern hemisphere (Luders and McMahon, 2006). The critical daylength of red pitaya was reported to be $\approx 12 \mathrm{~h}$, and the two switches between inductive and noninductive periods for red pitaya occur at the vernal and autumnal equinoxes, respectively (Jiang et al., 2012).

Southern Taiwan is located in the tropical region $\left(\approx 23.5^{\circ}-21.5^{\circ} \mathrm{N}\right)$ and its temperature and light intensity in the winter are adequate for pitaya growth. Off-season production can be induced by interrupting the length of the night with about $4 \mathrm{~h}$ of artificial lighting (NB) (Jiang et al., 2012). Consequently, in orchard management, shoots developed during winter flower in the inductive period (springsummer), whereas shoots that developed in

Received for publication 13 Nov. 2015. Accepted for publication 15 Feb. 2016.

${ }^{1}$ Corresponding author. E-mail: wendy@ntu.edu. tw. rested buds are of different sizes, with the larger ones located at the distal end of shoots. In general, these buds tend to respond more effectively to the NB treatment before December (personal observation). Therefore, we hypothesized that bud swelling could be associated with the number of bracteoles that progressively differentiated, and could be used as an indicator for bud development. Furthermore, the effectiveness of NB treatment in the off-season production of pitaya might be associated with the bud development stages. Thus, the effects of bud development with or without NB treatment between January and March were investigated.

\section{Material and Methods}

Plant material. Ten-year-old red pitaya 'Shih Hou Cyuan' planted in a commercial orchard in Pintung, southern Taiwan $\left(22^{\circ} 41^{\prime} \mathrm{N}, 120^{\circ} 31^{\prime} \mathrm{E}\right)$ was used. The clone was characterized as a red flesh and selfcompatible hybrid, a progeny derived from the cross between $H$. undatus and Hylocereus polyrhizus, and designated as Hylocereus sp. The pitaya plants were grown in rows $3 \mathrm{~m}$ apart on a single-wire trellis system $1.5 \mathrm{~m}$ high. Bamboo supports were installed every $0.5 \mathrm{~m}$ in the rows, and one plant was tied to each support. Each row was $35 \mathrm{~m}$ in length with 70 plants contained $600-720$ shoots. The temperature, daylength, and photosynthetic daily light integral (DLI, mol $\mathrm{m}^{-2} \cdot \mathrm{d}^{-1}$ ) in 2011 in Pingtung were obtained from a nearby station of the Taiwan Central Weather Bureau (Table 1). The daylength was defined as the time between sunrise and sunset.

Bud stage classification. The buds on each flowering shoot were first classified into four stages (0-3) according to the swelling of the areole base. About, 20-30 buds of each stage were randomly selected, and their width and height were measured and averaged for building the classification standard (Table 2).

Bud anatomical study. In this study, 100 shoots of autumn NB treated and non-NB treated were selected for tracking bud development. The floral buds on the selected shoots were removed and the dormant buds were classified into four stages (0-3) and labeled on 14 Oct. 2011. Ten labeled buds of each stage were sampled from the distal end of different shoots on 22 Oct. 2011, 30 Oct. 2011, 3 Nov. 2011, and 6 Nov. 2011. The sampled buds were fixed in a formalin acetic acid ethanol solution for 5-7 $\mathrm{d}$ and then
Table 1. Mean temperature, daylength, and photosynthetic daily light integral (DLI) in 2011 in Pingtung, Taiwan.

\begin{tabular}{llcccc}
\hline & & \multicolumn{3}{c}{ Month } \\
\cline { 3 - 5 } & & Janury & February & March & 14 Oct. to 10 Nov. \\
\hline \multirow{2}{*}{ Temperature $\left({ }^{\circ} \mathrm{C}\right)$} & Maximum & 22.9 & 26.8 & 26.8 & 31.6 \\
& Average & 17.3 & 20.1 & 21.3 & 26.4 \\
Daylength $(\mathrm{h})$ & Minimum & 13.6 & 15.4 & 17.3 & 23.0 \\
DLI $\left(\mathrm{mol} \cdot \mathrm{m}^{-2} \cdot \mathrm{d}^{-1}\right)$ & Average & 10.9 & 11.4 & 12.0 & 11.4 \\
\hline
\end{tabular}
having been exposed to adequate long daylength. Toward the end of September, the

early spring flower during the noninductive 2005). However, a promising year-round production schedule remains lacking becaur ar-round production is to be achieved.

cactus bud transformation, several bracteoles are differentiated before the formation in progressive bud swelling before florat is sufficient for the floral bud emergence red pitaya (Jiang et al., 2012). Because of apical dominance, numerous areoles, except

,


transferred into $70 \%$ alcohol. Samples were dehydrated using a tertiary-butanol series for $30 \mathrm{~h}$, infiltrated with paraffin wax for 4-5 d, and finally embedded in paraffin wax. Samples of 10 to $15-\mu \mathrm{m}$ thickness were longitudinally sectioned using a rotary microtome (Leica RM2125; Leica Biosystems, Germany). The sections were stained with periodic acid-Schiff
Table 2. Width and height of buds in the four stages in 'Shih Hou Cyuan' red pitaya.

\begin{tabular}{lcccr}
\hline & \multicolumn{4}{c}{ Bud stage $(\mathrm{mm})$} \\
\cline { 2 - 5 } & 0 & 1 & 2 & 3 \\
\hline Width & $1.8 \pm 0.04 \mathrm{~d}$ & $2.5 \pm 0.07 \mathrm{c}$ & $3.3 \pm 0.07 \mathrm{~b}$ & $4.0 \pm 0.07 \mathrm{a}$ \\
Height & $0.2 \pm 0.01 \mathrm{~d}$ & $0.5 \pm 0.03 \mathrm{c}$ & $1.1 \pm 0.05 \mathrm{~b}$ & $1.7 \pm 0.05 \mathrm{a}$ \\
\hline
\end{tabular}

Data are mean \pm SE (20-30 buds randomly selected from each stage).

Different letters in the same row represent significant differences between treatments. $P<0.05$ (analysis of variance and least significant difference).

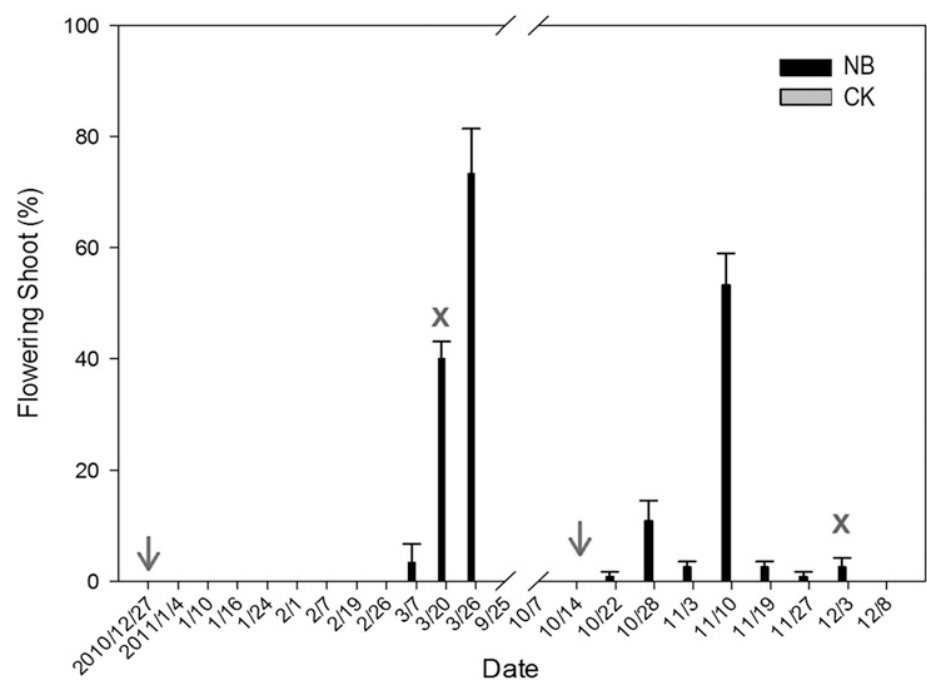

Fig. 1. The percentage of flowering shoots of 'Shih Hou Cyuan' red pitaya hybrid in response to the nightbreaking (NB) treatment in the noninductive period. The artificial lighting of NB treatments was provided daily (27 Dec. 2010 to 20 Mar. 2011) from 2100 to $0300 \mathrm{HR}$ within the experimental period. The " $\downarrow$ " and " $x$ " represented the beginning and end of the NB treatment, respectively.
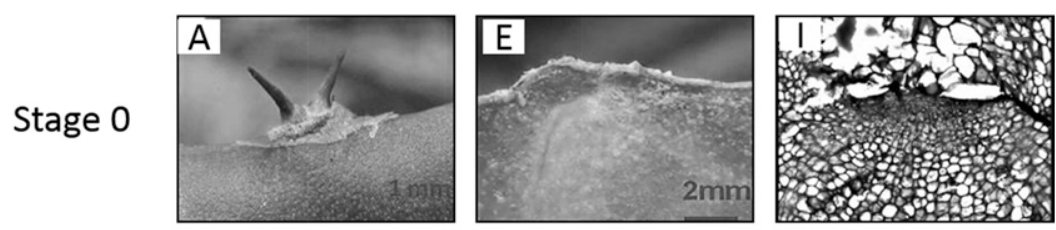

Stage 1
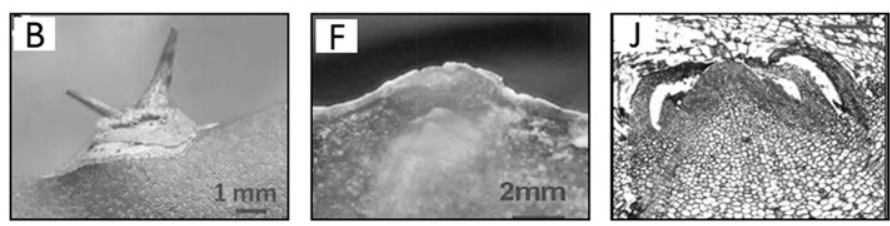

Stage 2
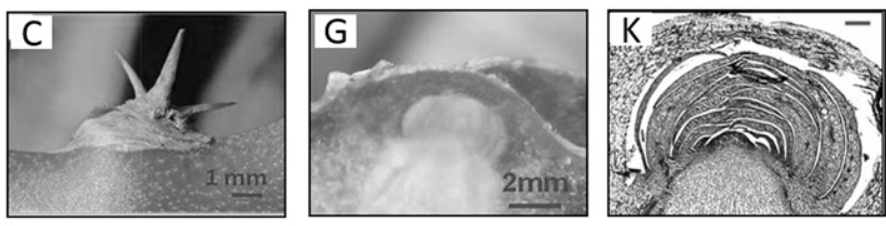

Stage 3
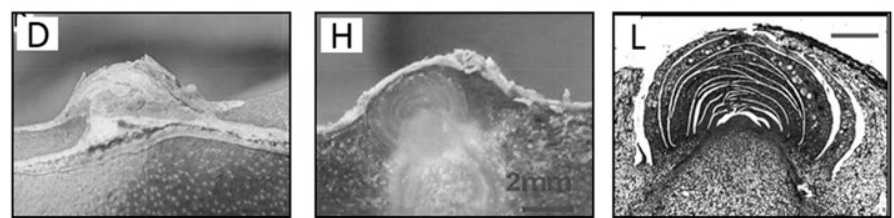

Fig. 2. Four bud stages on the shoots of 'Shih Hou Cyuan' red pitaya hybrid. Stage 0: (A, E, I); stage 1: $(\mathbf{B}, \mathbf{F}, \mathbf{J})$; stage 2: $(\mathbf{C}, \mathbf{G}, \mathbf{K})$; and stage 3: (D, H, L). Bar of I-L is $200 \mu \mathrm{m}$. reagent. All images were captured under an optical microscope (Nikon Alphaphot-2-YS2H; Nikon, Japan).

Winter NB treatment. The four stages $(0-3)$ of buds were classified and marked on 26 Dec. 2010. The NB treatment was applied from 27 Dec. 2010 to 20 Mar. 2011. Artificial lighting was provided from 2100 to $0300 \mathrm{HR}$ by $28-\mathrm{W}$ warm-white fluorescent lamps (EF3R28L-EX220; Grand Halo Technology Co. Ltd., Taiwan). The lamps were placed $30 \mathrm{~cm}$ above the plants at $1.5-\mathrm{m}$ intervals in lines located between every two rows, and the light intensity at the distal end of shoots was 2.4-5 $\mu \mathrm{mol} \cdot \mathrm{m}^{-2} \cdot \mathrm{s}^{-1}$ (measured using an LI250A light meter with an in-line LI-190SA quantum sensor; LI-COR, Lincoln, NE). The treatment was replicated five times, and each replicate contained 12 shoots on the outer canopy, and bud developing was tracked biweekly until 26 Mar. 2011. Sprouting and floral bud emergence were also recorded. Untreated plants were used as the control. The percentage of floral bud formation at each stage was recorded. The percentage of flowering and sprouting shoots as well as the average number of floral buds and new shoot per shoot were also recorded. For examining the positional effect on shoots, each shoot was divided into five equal-length sections from the distal to proximal ends. Sectional bud development and flowering percentage were obtained after dividing the number of flowers within each section by the total number of flowers.

$N B$ plus heating treatment. For the heating experiment, in 13 Jan. to 18 Feb. 2014, a small plastic house was constructed around the plants in the field for the NB + Heat treatment and the heating was achieved by two electric radiator heaters from 2000 to $0700 \mathrm{HR}$. The temperature inside and outside the plastic house was recorded by Hobo data logger $\left(\mathrm{HOBO}^{\circledR}\right.$ Pendant UA-001-64; ONSET, Bourne, MA). The three treatments were NB + Heat, NB alone, and Heat alone. Each treatment was replicated four times and 13 to 17 shoots labeled on the outer canopy per replication. Untreated plants were used as the control. The floral bud emergence was examined weekly until the end of the experiment.

Autumn NB treatment. The NB treatment was conducted from 14 Oct. 2011 to $12 \mathrm{Dec}$. 2011, and nontreated plants were used as the control. Lighting was applied as mentioned previously. The treatment was replicated five times and 12 shoots were labeled on the outer canopy per treatment. Floral buds on selected shoots were removed before beginning the experiment and flowering percentage and sprouting shoots were recorded biweekly.

Statistical analyses. CoStat 6.4 software (Costat 6.4, CoHort software) was used for statistically analyzing data. Resulting values were presented as means \pm SE of at least three units. The least significant difference test was used for comparing treatments when the analysis of variance showed significant differences between means. 


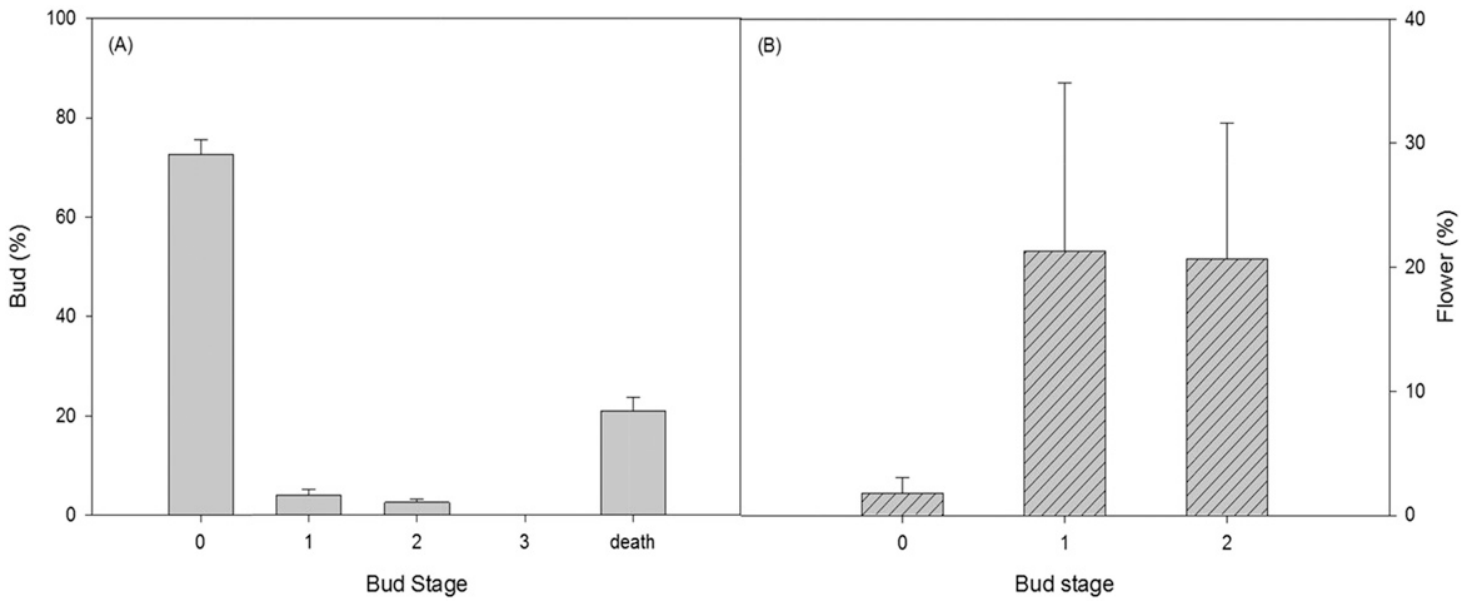

Fig. 3. (A) Percentage of each bud stage on 'Shih Hou Cyuan' shoots before the night-breaking (NB) treatment and (B) percentage of floral buds emerged from stage 0,1 , and 2 buds after undergoing 3 months of the NB treatment. The NB treatment was administered between 27 Dec. 2010 and 20 Mar. 2011.

\section{Results}

Flowering efficiency of 'Shih Hou Cyuan' in response to NB treatment in the noninductive period. The duration of NB treatment for flower differentiation was considerably longer in the winter season than in the autumn season (Jiang et al., 2012), and the chronicle record of floral bud emergence from the data set is graphed in Fig. 1. The durations of NB treatment to accumulate $88 \%$ of shoot flowering were 3 months during the winter (January-March) and 1 month for autumn. The floral buds of the winter NB treatment emerged during three specific dates $(7,20$, and 26 Mar. 2011), whereas emergence was much more dispersed over time during the autumn NB treatment.

Bud classification and transformation. The average width and length of the buds increased at higher stages (Table 2). Anatomically, the meristem underneath areoles transformed from a flat shape to dome shape and separated differentiating bracteoles at early developmental stages. In addition, the bracteole number increased as the stage number increased (Fig. 2).

In winter NB treatment, the initial bud stages recorded on 27 Dec. 2010 were $73 \%$, $4 \%, 2 \%$, and $0 \%$ for stages $0,1,2$, and 3 , respectively (Fig. 3A). Twenty-one percent of the buds were aborted and recorded as dead. After the remaining buds received 3 months of NB treatment, $20 \%$ of both stage 1 and 2 and $1.8 \%$ of stage 0 buds developed into floral buds (Fig. 3B). After the percentages were converted into numbers, we observed that $50 \%, 30 \%$, and $20 \%$ of the flowers were derived from stage 0,1 , and 2 buds, respectively (data not shown). Moreover, higher stage and dead buds were located more on the distal end of the shoots, where bud transformation was more active (Fig. 4).

Chronicle record of bud transformation in winter. Stage 1 and 2 buds could progress their development toward higher stages regardless of the NB treatment (Fig. 5). However, floral buds emerged only after the NB

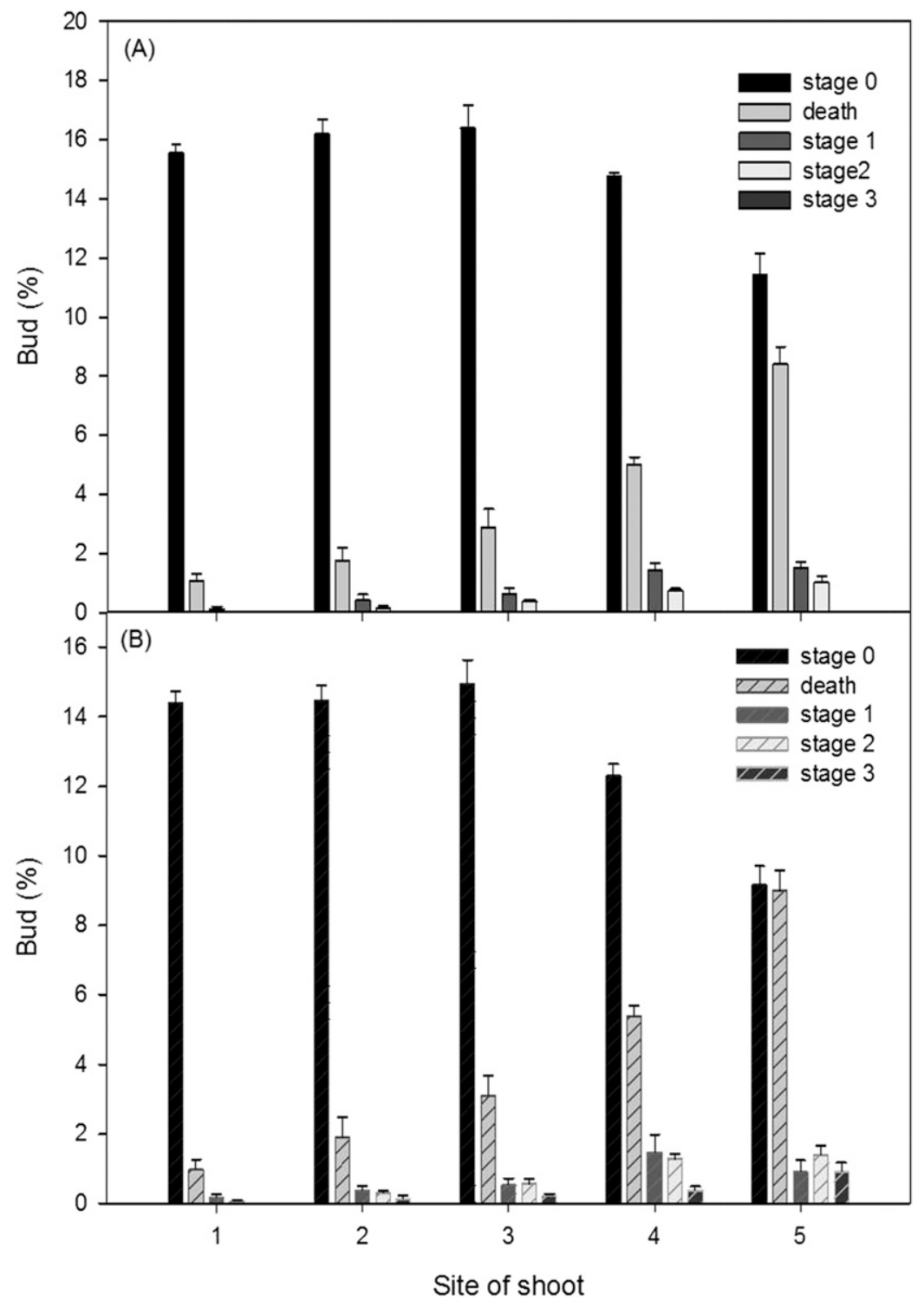

Fig. 4. Distribution of bud stages on each shoot of 'Shih Hou Cyuan' (A) before and (B) after the nightbreaking (NB) treatment. The NB treatment was administered between 27 Dec. 2010 and 20 Mar. 2011. The $X$ axis is the orientation of each shoot section, and numbers 1-5 represent the position of each section from the proximal to distal end. Data were collected from 12 shoots $(n=5)$. The sums of percentages of buds of all stages at all sections were $100 \%$. 
treatment, which doubled the bud transformations of stage 1 buds and advanced those of stage 2 buds by 1 month. After the buds

were transformed, the percentage of stage 1 buds toward stage 2 , stage 3 , floral, and dead buds were $\approx 40 \%, 20 \%, 30 \%$, and $10 \%$ on

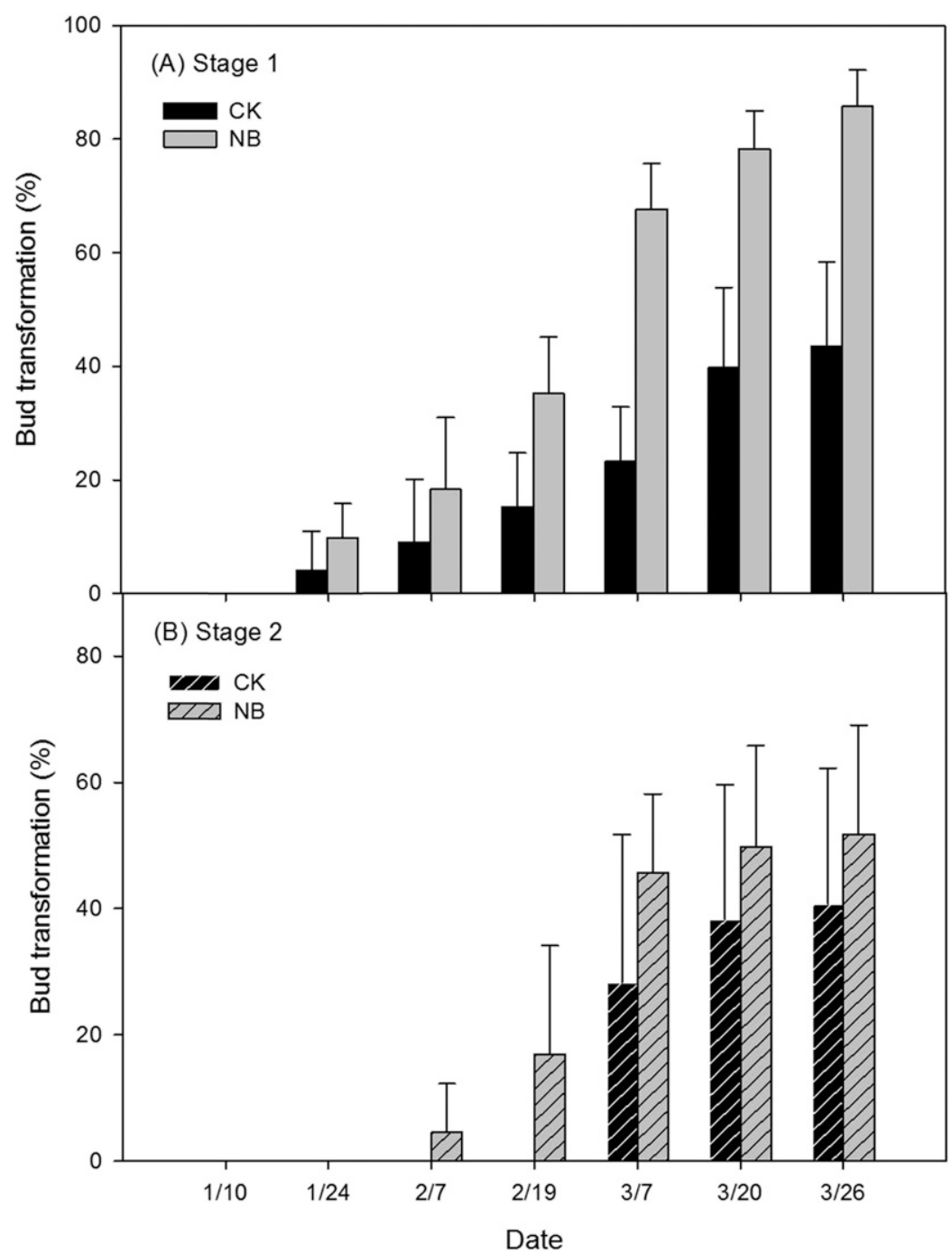

Fig. 5. Accumulated percentage of stage 1 and 2 buds on the shoots of 'Shih Hou Cyuan' that transformed into higher stage buds after 3 months of NB treatment. NB represents night-breaking treatment administered from 27 Dec. 2010 to 20 Mar. 2011. CK represents the control.
26 Mar. 2011, respectively (Fig. 6A). Of the $90 \%$ of stage 2 buds, $30 \%$ transformed to stage 3, 30\% died, and slightly less than $30 \%$ flowered (Fig. 6B). Under natural conditions, $50 \%$ of stage 1 and 2 buds developed one stage further by 26 Mar. 2011. The chronicle record of bud transformation revealed that most of the dead buds appeared before 7 Feb. 2011 and the abortion was more severe in the NB treatment of the stage 2 buds. In addition, bud transformation, regardless of the NB treatment, was accelerated after 7 Feb. 2011 (Fig. 6).

Anatomy of bud development. Bracteole differentiation was unaffected by NB treatment from 14 Oct. to 3 Nov. 2011. However, stage 3 buds did not emerge as floral buds, most died by 6 Nov. 2011 (Figs. 7 and 8). Three weeks were required for stage 0 and 1 buds to develop one stage further (Fig. 8A$\mathrm{H})$. Bud transformation from stage 2 to 3 was slightly faster (Fig. 8I-L), and the newly developed stage 3 buds required another week before emerging as floral buds (data not shown). The halted stage 3 buds required 3 weeks for emerging as floral buds (Fig. 8M-P).

\section{Discussion}

Because red pitaya is a long-day plant, its flowering is sensitive to daylength, and the daylength at the vernal and autumnal equinoxes is suggested to be of critical length for the flowering of red pitaya (Jiang et al., 2012). After the completion of floral induction, the majority of the buds underneath the areoles on the distal end of the matured shoots would not emerge as floral buds unless the apical dominance was removed (Borchert et al., 2005). Therefore, the percentage of flowering shoots could reflect yield instead of number of flowers. Besides, the flowering shoots were suggested not over $50 \%$ to yield larger fruits (personal observation).

After the autumnal equinox, the dormant buds were unable to develop floral buds before the subsequent vernal equinox, and
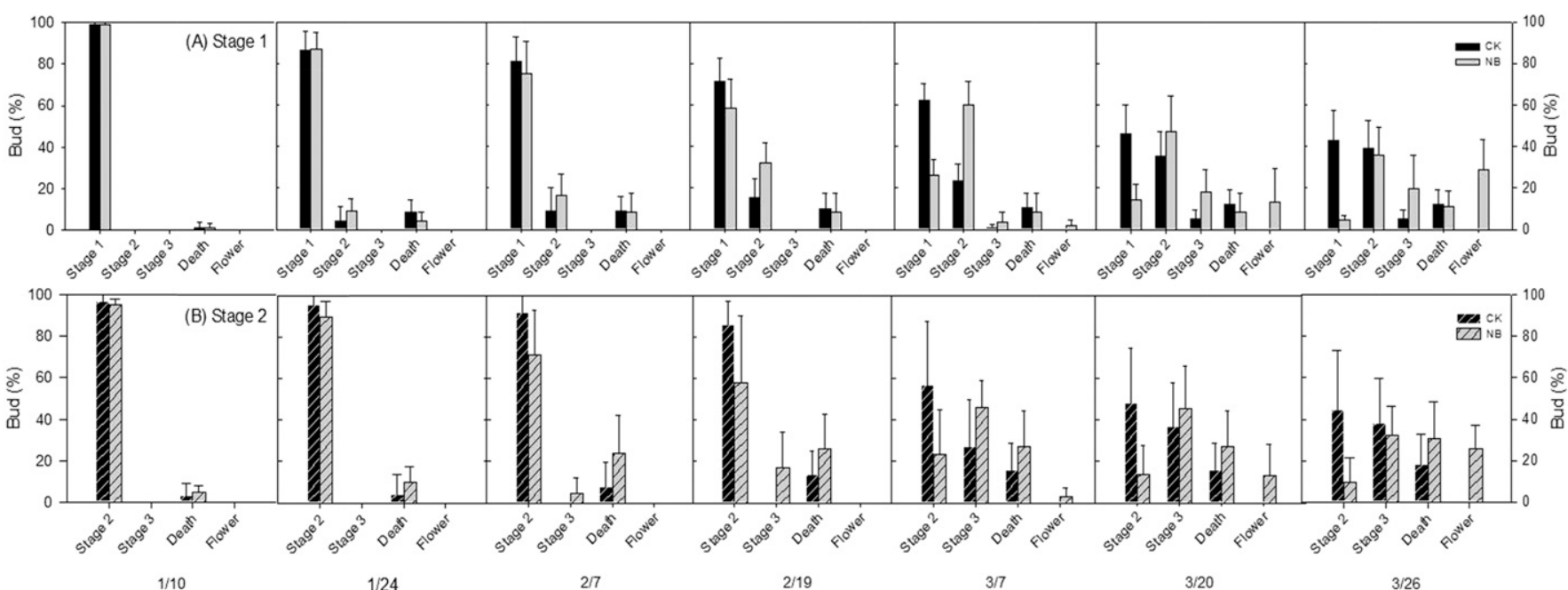

Fig. 6. Chronicle record of bud transformation of stage 1 and 2 buds after the night-breaking (NB) treatment and control in winter. The $X$ axis represents bud stages: stage 1, 2, 3, and dead floral buds. NB represents NB treatment that was administered from 27 Dec. 2010 to 20 Mar. 2011 and CK represents the control. 
some of them did not survive the cold season. Induced but dormant buds may develop into floral buds if the NB treatment is applied in the noninductive period (Jiang et al., 2012). In the trials of 2011, the duration of NB treatment for obtaining the first wave of off-season flowering was 3 -fold longer during the winter than during the autumn (Fig. 1).

In cacti, the apical meristem of the axillary bud produces many bracteoles before it turns into a floral primordium (Almeida et al.,

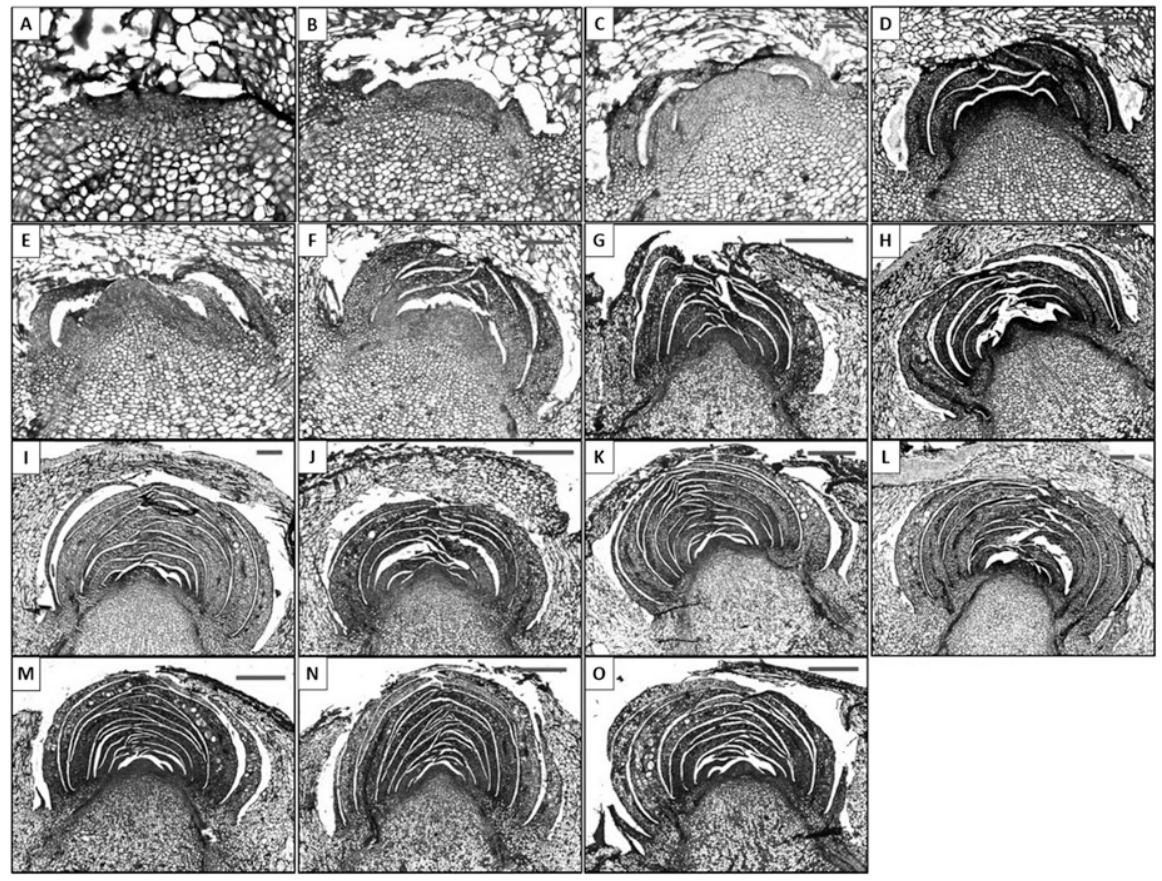

Fig. 7. Longitudinal sections of developing buds at different stages in the short daylength environment. Buds were classified and labeled on 14 Oct. 2011 and sampled on 22 and 30 Oct. and 3 and 6 Nov. 2011. A-D, $\mathbf{E}-\mathbf{H}, \mathbf{I}-\mathbf{L}$, and $\mathbf{M}-\mathbf{O}$ represent the development of stage $0,1,2$, and 3 buds, respectively. Bar length: $200 \mu \mathrm{m}$.

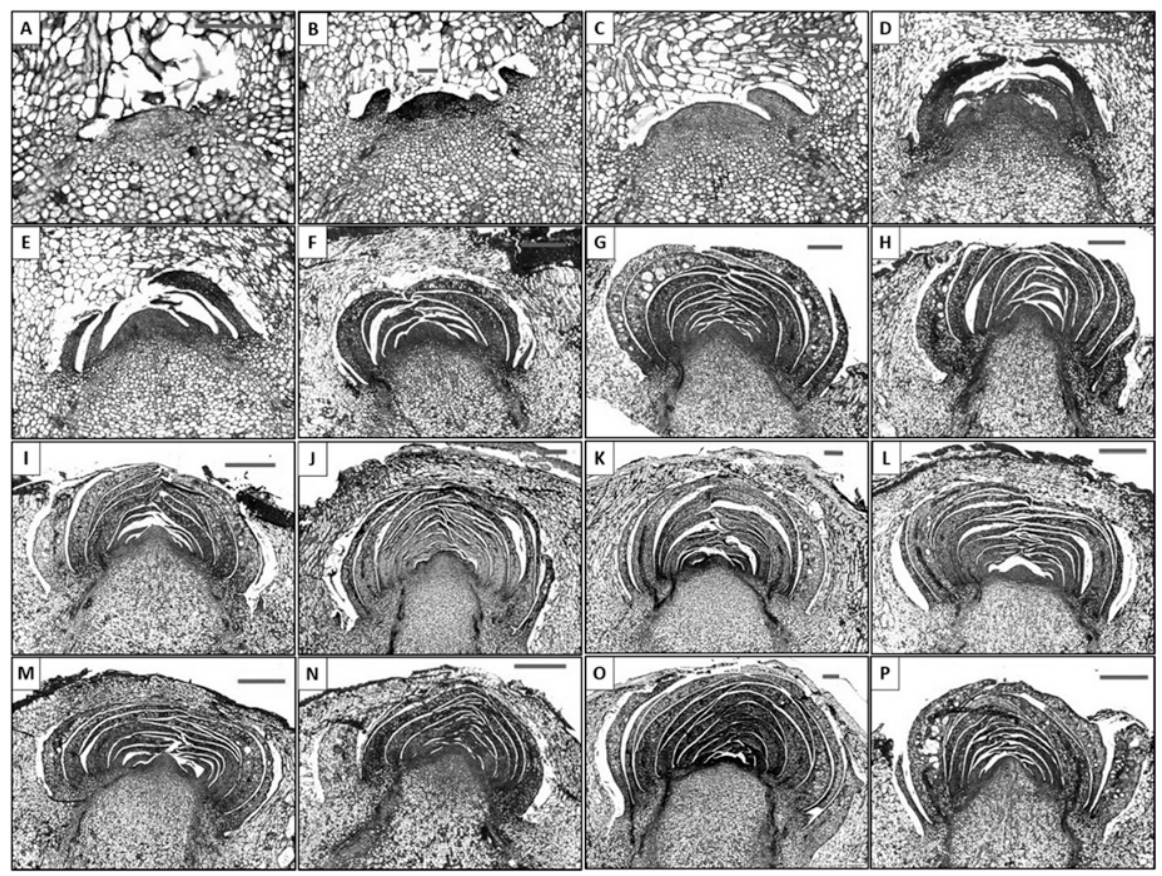

Fig. 8. Longitudinal sections of developing buds at different stages administered with the night-breaking treatment. Buds were classified and labeled on 14 Oct. 2011 and sampled on 22 and 30 Oct. and 3 and 6 Nov. 2011. A-D, E-H, I-L, and $\mathbf{M}-\mathbf{O}$ represent the development of stage 0, 1, 2, and 3 buds, respectively. $\mathbf{P}$ represents the floral bud. Bar length: $200 \mu \mathrm{m}$.
2010). Therefore, the size of buds is strongly associated with the number of bracteoles that progressively develop (Fig. 2; Table 2). In addition, bud transformation was observed throughout the shoots but was more active at the distal end, which is consistent with the flowering habit of pitaya (Fig. 4). Bud transformation toward higher stages did not cease in the noninductive period, suggesting that a long daylength was unnecessary for bracteole differentiation, although the differentiation rate was more rapid during the autumn than during the winter. The autumn temperature in Pingtung was adequate for pitaya growth (Table 1), which might explain why the bracteole differentiation rate did not change despite the decreasing daylength (Figs. 7 and 8).

The bud development and flowering of a given pitaya plant was proposed to depend on temperature, photoperiod, and irradiance (Jiang et al., 2011, 2012; Khaimov and Mizrahi, 2006; Khaimov et al., 2012; Luders and McMahon, 2006; Yen and Chang, 1997). In H. undatus, the DLI for obtaining optimal $\mathrm{CO}_{2}$ uptake was between 15 and $25 \mathrm{~mol} \cdot \mathrm{m}^{-2} \cdot \mathrm{d}^{-1}$ (Nobel and De La Barrera, 2004), suggesting that DLI in the winter of Pingtung might not be a limiting factor for growth and development (Table 1). Therefore, temperature likely plays a major role in the efficiency of off-season production. The chronicle record of bud death and acceleration of bud transformation suggest that major transitions in the environmental conditions were between 7 Feb. 2011 and 19 Feb. 2011. In Pingtung, the day temperature between 27 Dec. 2010 and 26 Mar. 2011 was more than $20{ }^{\circ} \mathrm{C}$, and the night temperature was considerably lower than the day temperature and variable (Table 1). In general, the night temperature was seldom lower than $15^{\circ} \mathrm{C}$ after $16 \mathrm{Feb}$. 2011 , which is consistent with the timing of the accelerated bud transformation. To verify our observation, 4 weeks of NB treatment resulted in $50 \%$ shoots flowered when night temperatures were maintained higher than $15{ }^{\circ} \mathrm{C}$ (Fig. 9). Therefore, we concluded that elevating night temperature along with NB could assure off-season production in deep winter in southern Taiwan for Hylocereu sp.

In southern Taiwan, a schedule of autumn off-season flowering by using NB treatment at about mid-October has been practiced for years. This schedule generally results in the first wave of abundant floral buds within 4-5 weeks of treatment. For example, NB treatment from 14 Oct. 2011 resulted in floral bud emergence first as a minor wave on 28 Oct. 2011 and a major wave on 10 Nov. 2011. The time required for developing into floral buds suggested that the buds might have developed from stage 3 and 2 buds. Furthermore, higher stage buds were found more sensitive to CI than lower stage buds, and most of the dead buds appeared in the lower temperature period between 10 Jan. and 7 Feb. 2011 (Fig. 6). The surviving buds actively transformed into floral buds within 6-7 weeks, 

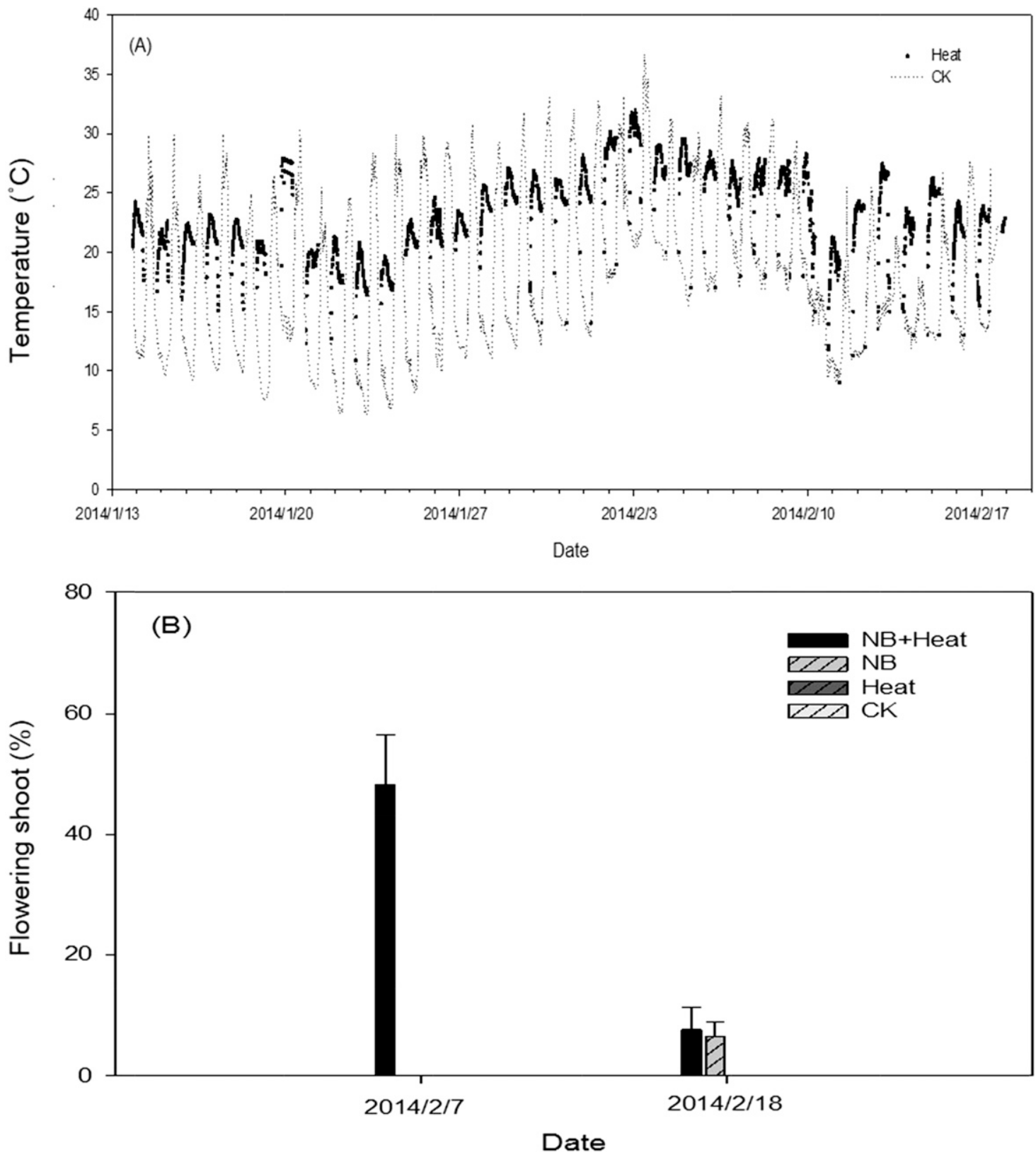

Fig. 9. Effect of heating on off-season flower in winter of 2014: (A) temperature recording during the experimental period, and (B) percentage of shoots flowering. Treatments were applied since $13 \mathrm{Jan} .2014$. NB represents night-breaking treatment and heat means night temperature elevation. (.) represents the daily temperature and ( $\bullet$ ) represents the night temperature of the heating treatment. CK represents the control.

from 19 Feb. 2011 to 26 Mar. 2011, indicating that the bud developing rate was similar in late spring and in autumn in southern Taiwan. Because the majority of floral buds emerged from stage 0 and 1 buds, the pattern of flowering dates was more concentrated.

Off-season flowering in red flesh pitaya is similar to natural flowering of yellow pitaya (Selenicereus megalanthus) in the winter of southern Taiwan (Jiang et al., 2011). The shoots sprouting in spring may be used for flower induction in the winter, whereas shoots sprouting in winter flower in the subsequent autumn (Jiang, 2005). Bracteole differentiation preceding floral bud emergence resulted in swollen buds and the size of the swollen buds was associated with bud stages. The higher the bud stage, the more effective the bud's response to NB treatment and more sensitive to CI it was. In the autumn off-season production trial, floral buds emerged from higher stage buds, whereas, the majority of floral buds emerged from lower stage buds. Low night temperature may limit bud development during the winter. Therefore, $\mathrm{NB}$ treatment should be applied along with night temperature elevation or after midFebruary when the minimum night temperature is increasing.

\section{Literature Cited}

Almeida, O.J.G., A.A.S. Paoli, and L.A. de Souza. 2010. Flower morpho-anatomy in Epiphyllum phyllanthus (Cactaceae). Rev. Mex. Biodiver. 81:65-80.
Borchert, R., S. Renner, Z. Calle, D. Navarrete, A. Tye, L. Gautier, R. Spichiger, and P. von Hildebrand. 2005. Photoperiodic induction of synchronous flowering near the equator. Nature 433:627-629.

Chang, T. 2003. Yield and quality of pitaya (Hylocereus undatus Britt. \& Rose) as affected by fruiting regulation. MS thesis, Natl. Chung Hsing Univ. Taiwan.

Hsu, W. 2004. Investigations on culture, growth habits and phenology in pitaya (Hylocereus spp.). MS Thesis, Natl. Taiwan Univ., Taiwan.

Jiang, Y. 2005. Classification, flowering and fruiting characteristics, and pruning of climbing cactus. MS Thesis, Natl. Taiwan Univ., Taiwan.

Jiang, Y., T. Lin, C. Lee, C. Yen, and W. Yang. 2011. Phenology, canopy composition, and fruit quality of yellow pitaya in tropical Taiwan. HortScience 46:1497-1502.

Jiang, Y., Y. Liao, T. Lin, C. Lee, C. Yen, and W. Yang. 2012. The photoperiod-regulated bud 
formation of red pitaya (Hylocereus sp.). HortScience 47:1063-1067.

Khaimov, A. and Y. Mizrahi. 2006. Effects of daylength, radiation, flower thinning and growth regulators on flowering of the vine cacti Hylocereus undatus and Selenicereus megalanthus. J. Hort. Sci. Biotechnol. 81:465-470.

Khaimov, A., O. Novak, M. Strnad, and Y. Mizrahi. 2012. The role of endogenous cytokinins and environmental factors in flowering in the vine cactus Hylocereus undatus. Isr. J. Plant Sci. 60:371-383.

Luders, L. and G. McMahon. 2006. The pitaya or dragon fruit (Hylocereus undatus). Agnote 778, No. D42. Northern Territory Government.

Nobel, P.S. and E. De La Barrera. 2004. $\mathrm{CO}_{2}$ uptake by the cultivated hemiepiphytic cactus, Hylocereus undatus. Ann. Appl. Biol. 144:1-8.

Yen, C. and F. Chang. 1997. Forcing pitaya (Hylocereus undatus Britt. \& Rose) by chemicals, controlled day length and temperature. Proc. Symp. Enhancing Competitiveness of Fruit Ind., Taichung District Agricultural Improvement Station, Taiwan. $3: 163-170$. 